\title{
Desarrollo turístico con enfoque sistémico, un reto hacia la sostenibilidad, valle de Vilcabamba.
}

\section{Tourism development with a systemic approach, a challenge towards sustainability, Vilcabamba Valley.}

\begin{abstract}
Mgtr. Gustavo Vladimir Paladines., ${ }^{1}$ Mgtr. Jenny Elizabeth Suárez Velasco., ${ }^{2}$ \& Dr. C. Eros Salinas Chávez. ${ }^{3}$
\end{abstract}

\section{Resumen.}

Ecuador está interesado en potenciar del desarrollo turístico, Vilcabamba posee un aumento de turistas notable lo que ha traído consigo oferta de productos y servicios turísticos sin un control técnico adecuado, para la solución de este problema se tuvo como objetivo, demostrar la validez de un desarrollo turístico con enfoque sistémico que permita sostenibilidad. La investigación se desarrolló partir de un enfoque cualicuantitativo de tipo descriptiva, exploratoria de campo, se concluyó que el modelo de Sergio Molina es un conjunto de procedimientos relacionados e integrados entre sí, que al lograr un enfoque sistémico en sus elementos se alcanza una perspectiva holística más centrada en lograr un desarrollo turístico sostenible, donde la población receptora juega un papel fundamental, además lograr la integración de los elementos del sistema turístico contribuirá al bienestar de la parroquia, un empoderamiento de la comunidad hará posible un desarrollo ordenado y racional.

Palabras claves: turismo, enfoque sistémico, sostenibilidad.

\begin{abstract}
.
Ecuador is interested in promoting tourism development, Vilcabamba has a remarkable increase in tourists which has brought with it tourism products and services without adequate technical control, for the solution of this problem was aimed at demonstrating the validity of a development tourism with a systemic approach that allows sustainability. The research was developed from a qualitative-quantitative approach of a descriptive,

\footnotetext{
${ }^{1}$ Universidad de las Fuerzas Armadas., Departamento de Ciencias Económicas, Administrativas y de Comercio (CEAC). Sangolquí, Pichincha, Ecuador. gvpaladines@espe.edu.ec

${ }^{2}$ Universidad de las Fuerzas Armadas., Departamento de Ciencias Económicas, Administrativas y de Comercio (CEAC). Sangolquí, Pichincha, Ecuador. jesuarez1@espe.edu.ec ${ }^{3}$ Universidad de La Habana, Facultad de Turismo. La Habana, Cuba. esalinas@ ftur.uh.cu
} 
exploratory field, it was concluded that the Molina model is a set of related and integrated procedures, that achieving a systemic approach in its elements achieves a holistic perspective more focused on achieving a sustainable tourism development, where the host population plays a fundamental role, also achieve the integration of the elements of the integrated tourism system will contribute to the welfare of the parish, an empowerment of the community to see economic results which will be sustainable.

Keywords: tourism, systemic approach, sustainability.

\section{Introducción}

Según la Organización Mundial del Turismo (OMT) "El turismo es un fenómeno social, cultural y económico relacionado con el movimiento de las personas a lugares que se encuentran fuera de su lugar de residencia habitual por motivos personales o de negocios/profesionales. Estas personas se denominan visitantes (que pueden ser turistas o excursionistas; residentes o no residentes) y el turismo tiene que ver con sus actividades, de las cuales algunas implican un gasto turístico" [1].

En la bibliografía internacional aparecen otras definiciones sobre el turismo, cercanas o similares, tan numerosas como son también los textos que abordan el tema, sin embargo, lo verdaderamente esencial es como todos los conceptos coinciden en que el turismo es un fenómeno socioeconómico, de marcada motivación psicosocial, que consiste en el desplazamiento de personas de lugares diferentes de sus sitios habituales de residencia, durante determinados períodos de tiempo $[2 ; 3]$

Ecuador es un país megadiverso interesado en la potenciación del desarrollo turístico, para lo cual apuesta a su variado patrimonio natural [4]; sus características biodiversas responden a diversos factores, entre ellos su ubicación en la línea equinoccial, la influencia de la corriente marina caliente del norte y de la corriente fría de Humboldt, la presencia de la cordillera de los Andes es otro factor que determina marcados pisos altitudinales y diferentes climas, la conjugación de estos factores demarca varias áreas biogeográficas con gran potencialidad e interés turístico [5;6;7].

El turismo en Ecuador se considera una de las actividades más significativas de la economía, que logró dinamizar en 2016, 285.5 millones de dólares solo en turismo interno, con más de 1.075 millones de dólares en ingresos solo en los primeros tres trimestres del año, el turismo constituye la tercera fuente de ingresos no petroleros detrás del banano y camarón, contribuyendo de manera directa con el 2,1\% del PIB nacional y de manera indirecta con el 5,1\%; además de ser un sector de gran dinamismo en el desarrollo social y capacidad de desarrollo en el ámbito local, ha estado ligado a este proceso y cambio de tendencia hacia la ecología y lo natural [8].

Con un peso importante en esta estrategia de potenciación del turismo, se encuentra al sur del país la provincia de Loja, que aporta una rica tradición en las artes, la música y otras expresiones de la cultura del Ecuador, además de extensas riquezas naturales [9]. Al Cantón Loja pertenece la Parroquia de Vilcabamba, ubicada a $40 \mathrm{kms}$ al sur oriente de la ciudad de capital de la provincia, uno de sus atractivos más publicitados es Vilcabamba, su nombre proviene de los vocablos quichuas (vilca $=$ sagrado, bamba $=$ valle), que significa "valle sagrado", donde anualmente se reciben varias inversiones de empresarios extranjeros $[10,11]$, 
Dentro de las riquezas naturales de esta parroquia se encuentran sus aguas a las que se les adjudican propiedades que mejoran la inmunidad para las enfermedades del corazón y favorecen la longevidad [12], según estudios censales locales sus habitantes pueden vivir más de 100 años y mantener la actividad física y laboral como cualquier adulto sano [13], estas características especiales hacen que el Valle de Vilcabamba sea "el único lugar en el mundo en donde es posible dar más años a la vida y más vida a los años” según el criterio del Dr. David Davies, científico inglés [14],todo esto le da la posibilidad de convertirse en potencial turístico zonal, regional e internacional.

En Vilcabamba siendo uno de los lugares más visitados del país, por turistas nacionales y extranjeros que llegan atraídos por su belleza natural, fiestas cívicas, fiestas religiosas, feriados, productos tradicionales y por la longevidad de sus habitantes, no existe una infraestructura hotelera organizada y centrada en el beneficio económico de sus habitantes, el cantón o la provincia; la gestión hotelera sigue un patrón de hosterías familiares, muchas veces administradas desde el extranjero que vierten sus dividendos de forma subterránea en arcas foráneas $[15,16,10]$.

El aumento de turistas en el Valle de Vilcabamba es notable y esto ha traído consigo situaciones como: oferta de productos y servicios turísticos sin un control técnico adecuado, incremento de los precios de los terrenos, extranjerización y descampesinización de propiedades en la zona urbana y rural desplazando a los pobladores especialmente a los longevos a las montañas, y otros sitios más agrestes y de difícil acceso $[15,17]$.

Este conjunto de consideraciones y las relaciones causa-efecto que llevan implícitas, propiciaron el planteamiento del problema científico de la investigación: ¿Cómo lograr un desarrollo turístico con enfoque sistémico para el desarrollo del turismo y la recreación en el Valle de Vilcabamba,?

Lo anterior permite considerar como objeto de estudio el Desarrollo Turístico-Recreativo y dentro del mismo de manera más concreta, un desarrollo turístico con enfoque sistémico para el desarrollo sostenible del turismo y la recreación en el Valle de Vilcabamba (Valle de Longevidad), lo que constituyó el campo de acción de esta investigación. Para aportar nuevos conocimientos a la solución del problema planteado se tiene como objetivo: Demostrar la validez de un desarrollo turístico con enfoque sistémico para lograr un desarrollo turístico-recreativo en el Valle de Vilcabamba.

\section{Métodos.}

La investigación se desarrolló partir de un enfoque cuali-cuantitativo de tipo descriptiva, exploratoria de campo, para su adecuada organización se desarrollaron las siguientes etapas o fases, etapa I: Diagnóstica, donde se realizó revisión bibliográfica, análisis y revisión de modelos teóricos de sistemas turísticos, inventario turístico de la parroquia y análisis de experiencias e identificación de estrategias exitosas para el desarrollo del turismo rural, etapa II: Proceso o ejecución donde se realizó el diseño de instrumentos para la obtención de información primaria, validación de instrumentos y la etapa III: Propuesta, confección y realización de FODA, confección de estrategias y plan de acción.

Para obtener la información primaria en la parroquia de estudio se diseñó una encuesta, que fue validada a través de expertos en turismo y recreación, vinculados a 
investigaciones, utilizando el método Delphi, con la finalidad de garantizar que los instrumentos abordaran de forma exhaustiva el problema investigado. Se les envió a 20 expertos por correo electrónico un documento explicativo de los objetivos y propósitos de la investigación, de su contribución y papel dentro de la misma, del grupo en que participaban, de las características del método Delphi como valoración grupal anónima y de estadística grupal. Se obtuvo de ellos el consentimiento para participar. En este documento, el experto evaluaba su propio nivel de competencia, además incluía otros datos generales que permitiría o no, su selección. Una vez recibida la respuesta de los expertos se realizaron los siguientes pasos:

a. Determinación del coeficiente de conocimiento $(\mathrm{Kc})$ del experto. Este coeficiente de conocimiento se calcula sobre la base de la autoevaluación presentada en escala creciente del 1 al 10, que incluye la apreciación de su calificación científica académica, sus años de experiencia y los resultados alcanzados en su labor profesional como experto y el conocimiento general que posee sobre las temáticas a valorar.

b. Cálculo del coeficiente de argumentación (Ka) o fundamentación, obtenido de la suma de los puntos a la respuesta del grado de influencia de las fuentes (análisis teóricos, consultas de trabajo de autores nacionales y extranjeros, su intuición basada en sus conocimientos y experiencias profesionales) que ha tenido para la excelencia en la ejecución de esta actividad.

c. Cálculo de la competencia a nivel de codificación de cada posible panelista como experto en el problema, objeto y campo en que se investiga mediante la fórmula:

$$
\mathrm{K}=1 / 2(\mathrm{Kc}+\mathrm{Ka})
$$

Una vez que se recibió la autoevaluación inicial de los candidatos a participar en la investigación y se realizó el cálculo del coeficiente de competencias de 11 expertos que respondieron.

Los expertos seleccionados obtuvieron un coeficiente de competencias mayor de 0.85 , considerado como alto y cumplieron además los criterios de inclusión y selección siguientes: ser profesionales vinculados.

A los mismos se les envió la encuesta, el instrumento inicial abarcó diez preguntas de las cuales se les preguntó la metodología en que estaban confeccionadas y preguntas con relación a aspectos a tener en cuenta para realizar estrategias óptimas para el desarrollo turístico recreativo. En la primera ronda siete expertos opinaron sobre la mejora de la metodología en que se debían hacer las preguntas, es decir estructura del diseño general de la encuesta, la estructura y contenido de cada pregunta y la interpretación de las mismas, por lo que fue necesario realizar modificaciones. El aspecto técnico relacionado con los criterios a tener en cuenta para el desarrollo turístico-recreativo cuatro expertos aportaron y sugirieron ser más específicos en las preguntas por lo que se tomaron en cuenta sus sugerencias.

Luego se pasó a la segunda ronda donde en relación a la cantidad de preguntas nueve expertos consideraron añadir una pregunta donde la comunidad pudiera opinar sobre su apreciación con el compromiso del estado en la tarea de las estrategias, por lo que de igual forma se realizaron modificaciones antes de pasar a la tercera ronda se modificaron 
algunos preguntas a partir de los criterios emitidos por los ellos, se tabularon los datos y se procesaron de forma tal, que en la siguiente ronda los participantes pudieron evaluar los resultados de la ronda anterior y valoraron nuevamente el contenido de cada pregunta.

En la tercera y última ronda se mostraron los argumentos unificados de las opiniones de los expertos en la ronda anterior, para que examinaran con más detalle cada pregunta y se les ofreció la posibilidad de dar una respuesta dicotómica, (de acuerdo ó no de acuerdo) con cada pregunta y así lograr un consenso de opiniones, quedando formada la encuesta por once preguntas, luego el instrumento fue sometido a una prueba piloto en un grupo de personas de la localidad que han tenido trabajo en el turismo del pueblo de Vilcabamba en años anteriores, para detectar problemas de aplicación de la encuesta, comprobar la comprensión de las preguntas y el funcionamiento del instrumento en relación al lenguaje o redacción de las preguntas y la facilidad de interpretación de cada una de ellas.

Finalmente, a la encuesta se le realizó el análisis de la fiabilidad mediante la prueba del alfa de Cronbach donde los resultados de cada pregunta fueron iguales o superiores a 0,893 en cada pregunta del instrumento. Los resultados anteriores demuestran la homogeneidad interna de la encuesta aportada por la contribución que cada ítem hace al instrumento en general.

Luego de toda esta validación, se aplicó una encuesta a pobladores del lugar y a turistas que se encontraban visitando el lugar en el periodo de trabajo de campo que se realizó en el área de estudio, se aplicaron 463 encuestas a pobladores y 387 a turistas, mediante el método de muestreo probabilístico intencional.

\section{Resultados}

Realizado el análisis y revisión de modelos teóricos de sistemas turísticos y analizar los resultados obtenidos en las encuestas aplicadas a pobladores y turistas nacionales e internacionales el investigador tomó como sostén teórico el modelo de Molina (2000), sin decir que sea mejor o peor que el de otros autores, el Sistema Turístico es un conjunto abierto de procedimientos relacionados e integrados con su medio ambiente en el que establece una serie de intercambios entre ellos. Posee un carácter dinámico y en constante cambio y adaptación. Su estructura es óptima cuando los subsistemas se organizan y establecen relaciones con el entorno admitiendo cambios y adaptaciones [18] .

Al respecto, Osorio (2000) señala que Molina "caracterizó a la actividad turística como un sistema abierto, con base en una interpretación de los modelos que formuló la Teoría General de Sistemas (TGS), desde una perspectiva holística (relacionado con el todo), que pretendía conocer la complejidad organizada y la totalidad. En este sentido, el turismo fue concebido de una manera totalizante e integradora, como "objeto orgánico", y el sistema turístico descrito como dinámico y probabilístico" [19].

Analizar al turismo como un sistema en el que se pueden definir sus partes y especificar vínculos entre ellas es muy apropiado, según el modelo de Sergio Molina, el sistema turístico se compone de 6 elementos que persiguen un objetivo en común:

Figura 1. Modelo de Sistema Turístico según Molina (2000) 


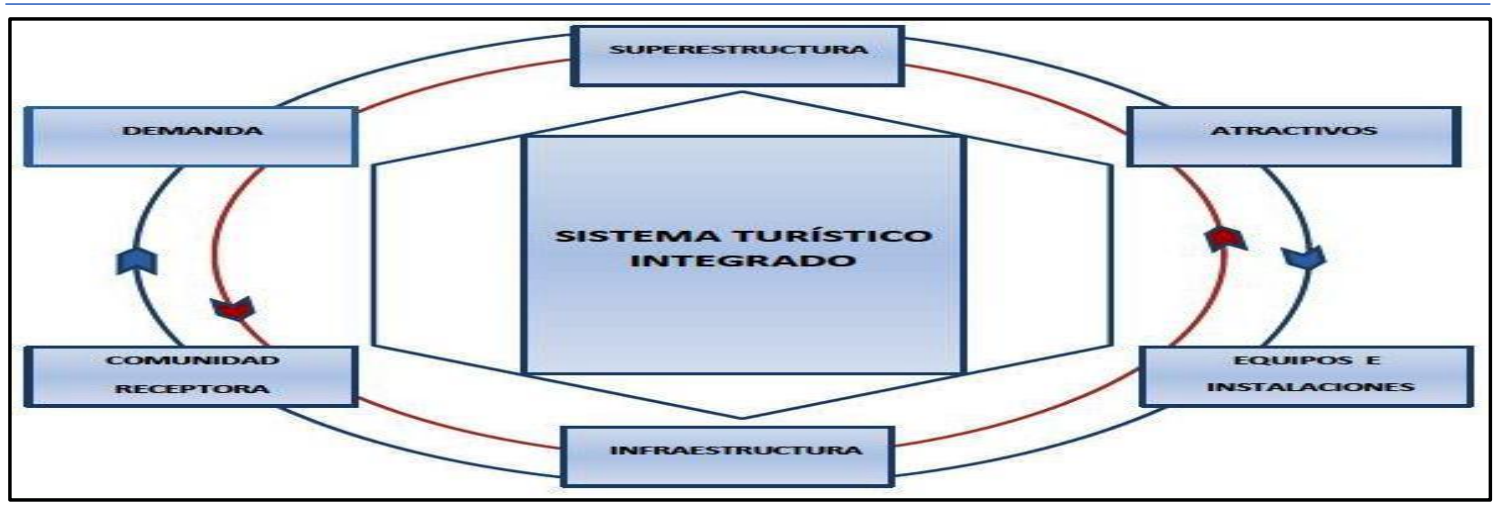

Fuente: Adaptación propia

Superestructura turística. Es el conjunto de instituciones públicas y/o privadas, que soportan jurídica y legalmente toda actividad turística, así como los procesos de mercadeo de dicho producto. Por ello se hace necesario considerar los soportes o bases legales en el ámbito turístico, partiendo en orden jerárquico, de la Constitución Política del Ecuador elaborada y aprobada en el 2008 hasta la base piramidal del Plan de Desarrollo y Ordenamiento Territorial de la parroquia Vilcabamba.

Comunidades receptoras. La comunidad local a pesar de tener sentido de pertenencia con la parroquia en cuanto a sus tradiciones e historia, presenta contrapeso con las opciones culturales exógenas de los migrantes residentes venidos de Norteamérica y Europa específicamente que han propiciado efectos aculturadores en los adolescentes y jóvenes contribuyendo a la pérdida de identidad cultural lo que requiere de una urgente intervención con capacitación, animación sociocultural que permita en la población local la consolidación de las pertenencias comunal, regional, histórica y cultural.

Las comunidades receptoras como parte del Sistema Turístico, son un elemento imprescindible en la gestión y planificación del turismo y por ello es necesario que su participación sea activa y consiente en el proceso de toma de decisiones consolidando así su sentido de pertenencia e identidad con el lugar de origen o habitable. En este sentido, la OMT (2015) sostiene que "Implantar conciencia turística" no significa convencer a la sociedad de que el turismo es maravilloso y una opción ideal para mejorar la situación económica de la comunidad. Implantar conciencia turística es ante todo informar sobre las oportunidades y riesgos que la actividad turística puede suponer para la comunidad y también preparar y formar a la sociedad para asumir dicha actividad evitando tensiones y maximizando los beneficios de todo tipo (económicos, sociales, ambientales, culturales, etc.). La participación de la sociedad en el proceso de desarrollo turístico es imprescindible desde un enfoque democrático y de equidad social de las actuaciones" [20].

En cuanto a organizaciones no gubernamentales, Vilcabamba cuenta con asociaciones civiles conformadas por miembros de la comunidad, entre ellas se destacan:

Asociación de Artesanas “Los Huilcos”, Asociación Artesanal de Productores Ecológicos de Café Especial, Asociación de Longevos de Vilcabamba, Asociaciones Deportivas: Clubs Deportivos: "La Pila”, “Alianza” y "Dinámicos”, Comité Integral Multicultural de Vilcabamba, Asociación de Mujeres de Vilcabamba (AMUVIL) Comité de Apoyo y Gestión por los Derechos de Vilcabamba. 
Actores Gubernamentales: Gobierno Autónomo Descentralizado Parroquial de Vilcabamba, Centro de Información y Facilitación Turística, entre otros

Demanda turística : Los sitios más visitados en el Ecuador según la Asociación Nacional de Operadores de Turismo Receptivo de acuerdo al segmento terrestre está en primer lugar Guayaquil (51,23\%), seguido de Quito (47,95\%), Cuenca (12,30\%), Otavalo $(11,48 \%)$, Machala (9,84\%), Ibarra y Salinas (43\%), Baños $(9,02 \%)$ entre los primeros; seguido de otros lugares de visitas se encuentran las ciudades como Manta $(5,74 \%)$, Amazonía, Esmeraldas y Bahía de Caráquez (4,10\%), Loja (3,69\%) y Vilcabamba $(0,82 \%)$, siendo así que Vilcabamba se encuentra dentro de las cifras turísticas en el Ecuador (OPTUR, 2013).

La demanda del turismo en Vilcabamba ha incrementado en los diferentes años anteriores tal como se muestra en el gráfico $\mathrm{N}^{\circ} 24$, se observa que en el 2010 el número de turistas fue de 12,486 personas (16,7\%), para el 2012 fueron 12, 699 personas (17\%), en el año 2014 fue de 10,140 personas $(13,61 \%)$ y para el 2016 fue de 12,947 personas $(17,38 \%)$, y hasta el mes de mayo 2017 el total de turistas registrados es de 5363 turistas de acuerdo a las estadísticas promovidas por el Centro de Facilitación de Información Turística Vilcabamba.

Los turistas que más visitan la parroquia son de Ecuador provenientes especialmente de Loja, Guayaquil, Quito, Machala y Cuenca, seguido de extranjeros de USA, Canadá, Suiza, Alemania, Francia, Argentina y España entre los más principales. La ocupación de los turistas es especialmente estudiantes de nivel instrucción superior cuyo objetivo de visita es por vacación, recreo y ocio, seguido de visita familiar, y al ser Vilcabamba un lugar turístico existe una gran variedad de comercio por la cual también el motivo principal es por negocio y motivos profesionales.

\section{Atractivos turísticos:}

A continuación, se presenta el inventario de Atractivos Turísticos u Oferta de Vilcabamba, basado en la Guía Metodológica para la Jerarquización de Atractivos y generación de espacios turísticos del Ministerio de Turismo de Ecuador (2017)

Tabla 1. Categoría: Sitios Naturales

\begin{tabular}{c|c|c|l}
$\begin{array}{c}\text { Nombr } \\
\text { e }\end{array}$ & Tipo & Subtipo & \multicolumn{1}{c}{ Descripción } \\
\hline $\begin{array}{c}\text { Parque } \\
\text { Pacional } \\
\text { s }\end{array}$ & Áreas \\
protegidas & $\begin{array}{c}\text { Parque } \\
\text { Nacional }\end{array}$ & $\begin{array}{l}\text { El Parque nacional Podocarpus está ubicado en las provincias de Loja y Zamora } \\
\text { Chinchipe, en el sur oriente del Ecuador. Es una zona de megadiversidad floral y } \\
\text { faunistica de alto grado de endemismo debido a su ubicación entre sistemas } \\
\text { biológicos diversos }\end{array}$ \\
\hline $\begin{array}{c}\text { Cerro el } \\
\text { Mandango }\end{array}$ & Montañas & $\begin{array}{c}\text { Baja } \\
\text { montañas }\end{array}$ & $\begin{array}{l}\text { El cerro El Mandango, originalmente significa 'Cara dura' o 'Venerable' y es } \\
\text { también llamado "Dios Acostado" porque desde lejos tiene la apariencia de una cara } \\
\text { que al parecer está recostado sobre la llanura Tiene una altitud de 1200 metros y } \\
\text { posee forma de una pirámide. }\end{array}$ \\
\hline $\begin{array}{l}\text { Lagunas } \\
\text { del } \\
\text { Compadre }\end{array}$ & $\begin{array}{l}\text { Ambientes } \\
\text { lacustres }\end{array}$ & Laguna & $\begin{array}{l}\text { Conforman un sistema lacustre integrado por 100 lagunas con gran valor escénico. } \\
\text { Forman parte del Parque Nacional Podocarpus sobre el nudo de Sabanillas en la } \\
\text { región del Picacho del Cóndor. }\end{array}$ \\
\hline
\end{tabular}




\begin{tabular}{l|l|l|l}
\hline $\begin{array}{c}\text { Laguna } \\
\text { Rabadilla de } \\
\text { Vaca }\end{array}$ & $\begin{array}{c}\text { Ambientes } \\
\text { lacustres }\end{array}$ & Laguna & $\begin{array}{l}\text { Se encuentra ubicada hacia el oeste, cerca de la división andina oriental al pie de un } \\
\text { acantilado originado por un gran glaciar del valle que se proviene de las elevaciones } \\
\text { más altas de la línea divisoria. }\end{array}$ \\
\hline $\begin{array}{l}\text { Estoraques } \\
\text { de } \\
\text { Yamburara } \\
\text { Alto }\end{array}$ & $\begin{array}{l}\text { Fenòmenos } \\
\text { geológicos }\end{array}$ & $\begin{array}{l}\text { Estoraque } \\
\text { o columnas } \\
\text { de tierra }\end{array}$ & $\begin{array}{l}\text { Son largas y altas columnas de tierra verticales centenarias producto de la erosión y } \\
\text { aún se mantienen intactas. Según los habitantes del lugar tienen poderes } \\
\text { sobrenaturales por lo que muchas personas consideran son señales naturales de } \\
\text { comunicación con seres extraterrestres. }\end{array}$ \\
\hline $\begin{array}{l}\text { Rios } \\
\text { capamaco, } \\
\text { yambala, } \\
\text { chamba, } \\
\text { uchima, } \\
\text { vilcabamb } \\
\text { a }\end{array}$ & Rios & Ríos & $\begin{array}{l}\text { Según investigaciones realizadas por los Dres. Gary Gordon (inglés) y Kokichi } \\
\text { Otain (japonés) se determinó que las aguas de los ríos poseen propiedades } \\
\text { medicinales por la presencia del magnesio y hierro lo que produce un efecto de } \\
\text { chelación", es decir, de diluir grasas, colesterol y minerales como el calcio. Esta } \\
\text { podría ser la razón del secreto de la longevidad en los habitantes del lugar. La } \\
\text { confluencia de los ríos Chamba y Uchima generan el río Vilcabamba }\end{array}$ \\
\hline $\begin{array}{l}\text { Agua de } \\
\text { hierro }\end{array}$ & $\begin{array}{l}\text { Aguas } \\
\text { subterráneas }\end{array}$ & $\begin{array}{l}\text { Manantial } \\
\text { de agua } \\
\text { mineral }\end{array}$ & $\begin{array}{l}\text { Fuente de aguas subterráneas que provienen de la Cordillera Oriental de los Andes } \\
\text { y pasan por el Valle de Vilcabamba. Posee propiedades nutritivas para la piel por } \\
\text { su composición rica en minerales como el hierro del cuál deriva su nombre. }\end{array}$ \\
\hline
\end{tabular}

Fuente: DPDT, MINTUR, 2016, Dirección de Planificación de Destinos

Turísticos 2016

Elaboración: adaptación y actualización propia

Tabla 2.Categoría manifestaciones culturales, acervo cultural y popular

\begin{tabular}{|c|c|c|c|}
\hline Nombre & Tipo & Subtipo & Descripción \\
\hline $\begin{array}{l}\text { Fiestas del } \\
\text { Sagrado } \\
\text { Corazón de } \\
\text { Jesús }\end{array}$ & $\begin{array}{l}\text { Acervo } \\
\text { cultural y } \\
\text { popular }\end{array}$ & $\begin{array}{c}\text { Fiestas religiosas, } \\
\text { tradiciones y } \\
\text { creencias populares }\end{array}$ & $\begin{array}{l}\text { Es una fiesta que se realiza en la última semana del mes de julio como } \\
\text { forma de agradecer al Sagrado Corazón de Jesús por los favores que } \\
\text { realiza no solo a la comunidad de Vilcabamba, sino las parroquias } \\
\text { aledañas. }\end{array}$ \\
\hline $\begin{array}{c}\text { Fiestas de } \\
\text { Parroquializaci } \\
\text { ón }\end{array}$ & $\begin{array}{l}\text { Acervo } \\
\text { cultural y } \\
\text { popular }\end{array}$ & $\begin{array}{c}\text { Fiestas religiosas, } \\
\text { tradiciones }\end{array}$ & $\begin{array}{l}\text { Es una festividad cívica en honor a la instauración de Vilcabamba como } \\
\text { Parroquia. Se realiza cada } 1 \text { de septiembre con actividades culturales, } \\
\text { deportivas, religiosas como Te Deum, Misas u otros oficios. }\end{array}$ \\
\hline $\begin{array}{c}\text { Fiestas en } \\
\text { honor al Señor } \\
\text { de la Buena } \\
\text { Esperanza }\end{array}$ & $\begin{array}{c}\text { Acervo } \\
\text { cultural y } \\
\text { popular }\end{array}$ & $\begin{array}{l}\text { Fiestas religiosas, } \\
\text { tradiciones }\end{array}$ & $\begin{array}{l}\text { La última semana de septiembre los priostes o cofradías de la parroquia } \\
\text { se unen para realizar las festividades con misas, oficios religiosos como } \\
\text { novenas. También se llevan a cabo vuelta ciclística de montaña desde el } \\
\text { parque central, una maratón de mujeres desde la entrada a Vilcabamba., } \\
\text { concurso de gallos, }\end{array}$ \\
\hline $\begin{array}{l}\text { Fiestas de la } \\
\text { Virgen de } \\
\text { Fátima y Señor } \\
\text { de la Justicia } \\
\text { de Izhcayluma }\end{array}$ & $\begin{array}{l}\text { Acervo } \\
\text { cultural y } \\
\text { popular }\end{array}$ & $\begin{array}{l}\text { Fiestas religiosas, } \\
\text { tradiciones }\end{array}$ & $\begin{array}{l}\text { Las fiestas se realizan en el barrio Izhcayluma con la procesión de las } \\
\text { imágenes desde el Hospital de Vilcabamba hasta la Capilla de Nuestra } \\
\text { Señora de Fátima donde se celebra la eucaristía al aire libre. También se } \\
\text { presentan espectáculos de juegos pirotécnicos, danzas, artistas y bailes } \\
\text { populares. }\end{array}$ \\
\hline $\begin{array}{c}\text { Feria } \\
\text { agroecológica } \\
\text { y orgánica }\end{array}$ & $\begin{array}{c}\text { Acervo } \\
\text { cultural y } \\
\text { popular }\end{array}$ & Ferias y mercados & $\begin{array}{l}\text { El último domingo, de cada mes se organiza la feria agroecológica en la } \\
\text { plaza, desde las 08:00 hasta las 16:00. }\end{array}$ \\
\hline Pacha feria & $\begin{array}{l}\text { Acervo } \\
\text { cultural y } \\
\text { popular }\end{array}$ & Ferias y mercados & $\begin{array}{l}\text { Es una feria artesanal que se realiza las segundas y terceras semanas de } \\
\text { cada mes en el parque central de la parroquia frente a la iglesia matriz. }\end{array}$ \\
\hline Comida Tipica & $\begin{array}{l}\text { Acervo } \\
\text { cultural y } \\
\text { popular }\end{array}$ & Gastronomía & $\begin{array}{l}\text { Entre la riqueza gastronómica de Vilcabamba se destacan: la sopa de } \\
\text { arveja con guineo, cecina de chancho, estofado de pollo, seco de chivo, } \\
\text { caldo de gallina criolla, cuero con papa, sancocho, menestras, el típico } \\
\text { café filtrado, mote, humitas, tamales y sango, repe, quimbolitos, cuy, } \\
\text { fritada, chicharrones, chanfaina, guatitas, chifles, tortilla de gualo. }\end{array}$ \\
\hline Dulcería & $\begin{array}{c}\text { Acervo } \\
\text { cultural y } \\
\text { popular }\end{array}$ & Gastronomía & $\begin{array}{l}\text { La dulcería típica vilcabambense se expresa en el quesillo con miel, } \\
\text { roscones, bizcochuelos, bocadillos, dulces y frutas pasadas en miel, } \\
\text { mazapanes, quesadillas lojanas, manjar, dulce de guayaba, alfeñiques o } \\
\text { melcochas, galletas y pasteles. }\end{array}$ \\
\hline Chamicos & $\begin{array}{c}\text { Acervo } \\
\text { cultural y } \\
\text { popular }\end{array}$ & Artesanía & $\begin{array}{l}\text { Es un cigarro tradicional, tipo habano, elaborado artesanalmente. Posee un } \\
\text { aroma especial y generalmente es elaborado por los ancianos de la } \\
\text { localidad. }\end{array}$ \\
\hline
\end{tabular}


Fuente: DPDT, MINTUR, 2016, Dirección de Planificación de Destinos

Turísticos 2016

\section{Discusión.}

Elaboración: adaptación y actualización propia.

Ante todo, lo explicado para describir y explicar la actividad turística del Valle de Vilcabamba, se recurre al enfoque sistémico, considerando cada elemento o subsistema con el fin de determinar su potencialidad.

Los componentes o subsistemas se presentan como instrumentos interrelacionados y transversalizados para revitalizar o repotenciar espacios concretos como el Valle de Vilcabamba, a partir de su dialéctica territorial, sociocultural, económica y política, Como algo intrínseco a su conceptualización, una propuesta sistémica de desarrollo turístico integrado debe contemplar las siguientes premisas:

Asumir la transversalidad en la integración de los subsistemas superando una posible limitación de estrategias dirigidas exclusivamente a los tradicionales renglones de ofertademanda turísticas.

Que se considere el carácter endógeno del desarrollo integrado como parte del desarrollo sostenible.

Hacer una lectura el territorio más que como un simple soporte de la actividad, como configurador de un desarrollo integrado.

Que los espacios sean considerados como un «sistema abierto» y en consecuencia expuestos a las influencias exógenas lo que les haga estar en constante transformación y evolución.

Que el desarrollo turístico integrado sea participativo, requiriendo involucrar a la sociedad en general como la comunidad receptora y los agentes tanto públicos como privados para alcanzar consensos que hagan posible el logro de objetivos comunes que permita enfocar estrategias y programas de forma coordinada y consensuada.

\section{Conclusiones}

> El análisis de algunos modelos teóricos de sistemas turísticos y específicamente el modelo de Molina, permitió una perspectiva holística más centrada en lograr un desarrollo turístico recreativo sostenible en el valle de Vilcabamba donde la población receptora juegue un papel fundamental en dicha tarea.

Lograr una sistematicidad entre los elementos de integran el sistema turístico integrado contribuirá a el bienestar de la parroquia de Vilcabamba, permitiendo un empoderamiento de la comunidad por ver resultados económicos lo cual hará sea sostenible 


\section{Referencias Bibliográficas}

[1] Organización Mundial del Turismo, «media.unwto.org,» s/n febrero 2017. [En línea]. Available: http://media.unwto.org/es/content/entender-el-turismo-glosariobasico. [Último acceso: 12 noviembre 2017].

[2] N. J. Medina Pérez , J. N. Santamarina Guerra y E. Salinas Chávez, Productos turisticos . Desarrollo y Comercialización, primera ed., E. d. A. E. d. H. y. Turismo, Ed., Habana: Ediciones Balcón, 2010, p. 3.

[3] Salinas, «Geografía y Turismo. Aspectos Territoriales del Manejo y Gestión del Turismo.,» Editorial Félix Varela, La Habana, 2013.

[4] Banco Interamericano de Desarrollo, «PLANDETUR-2025,» BID, Quito, 2012.

[5] K. Jaffe, La riqueza de las naciones: una visión interdisciplinaria, Caracas: Equinoccio, 2007.

[6] J. Llorente y J. J. Morrone, Introducción a la biogeografía en Latinoamérica: teorías, conceptos, métodos y aplicaciones, Biogeography, 2001.

[7] MINTUR, «Programa Nacional de Capacitación Turística,» Quito, 2009.

[8] Ecuador en Cifras, «Ecuador potencia turística,» 16 marzo 2017. [En línea]. Available: $\quad$ http://servicios.turismo.gob.ec/index.php/portfolio/turismocifras?layout=blog.

[9] EcuRed, «EcuRed,» 24 Marzo 2017. [En línea]. Available: https://www.ecured.cu/Provincia_Loja.

[10] Municipio de Loja, «Municipio de Loja,» 2017. [En línea]. Available: http://www.loja.gob.ec/contenido/vilcabamba. [Último acceso: febrero 2017].

[11] Secretaría Nacional de Planificación y Desarrollo, «Plan de Desarrollo y ordenamiento territorial. Parroquia Vilcabamba.Cantón Loja,» Vilcabamba, 2015.

[12] C. Samariego y G. Laspina, «Migracion Residencial en el valle de Vilcabamba y su impacto socio espacial,» PUCE, Loja, 2015.

[13] A. Leaf y et al, Vilcabamba. The secret valley of the centenarian, 2nd ed., San Francisco: New Wisdom Press, 2013.

[14] D. Davies, The Centenarians Of The Andes, 2nd ed., Edition in Books, 2013.

[15] M. Crepo, «Extrangerización de la tierra agrícola,» 2014. 
[16] M. Hayes, «Una nueva migración económica : el arbitraje geográfico de los jubilados estadounidenses hacia los países andinos,» Boletin Andina Migrante, pp. 2-13, 2013.

[17] J. Gascón y E. Cañada, Turismo residencial y gentrificación rural, Tenerife: PASOS, 2016.

[18] S. Molina , Conceptualización del Turismo, 4 ed., N. Editores, Ed., Mexico DF: Limusa, 2003.

[19] M. Osorio García, «Nuevos Caminos para el Estudio del Turismo desde la Teoría de Sistemas,» Convergencia. Revista de Ciencias Sociales, vol. 7, n 23, pp. 218235, Septiembre 2000.

[20] OMT, «El turismo y los Objetivos de Desarrollo Sostenible,» UNWTO, 2015.

[21] T. Luzuriaga, «Análisis de la ocupación hotelera de la ciudad de Loja, 2009,» Universidad Técnica Particular de Loja, Loja, 2010.

[22] D. Cabascango y P. Guamán, Tierras para Miel y Bosques. Estudio de caso, Vilcabamba, Loja, 2015.

[23] AME, Asociación de Municipalidades del Ecuador, 2007.

[24] Banco Interamericano de Desarrollo (BID), «PLAN ESTRATÉGICO DE DESARROLLO DE TURISMO SOSTENIBLE PARA ECUADOR PLANDETUR 2020,» S/N, Quito, 2012.

\section{UCiencia}




\section{Para citar el artículo indexado.}

Paladines G., Suárez J. \& Salinas E. (2018). Desarrollo turístico con enfoque sistémico, un reto hacia la sostenibilidad, valle de Vilcabamba. Revista electrónica Explorador Digital 2(3), 24-35. Recuperado desde:

http://cienciadigital.org/revistacienciadigital2/index.php/exploradordigital/article/view/334/7 $\underline{46}$

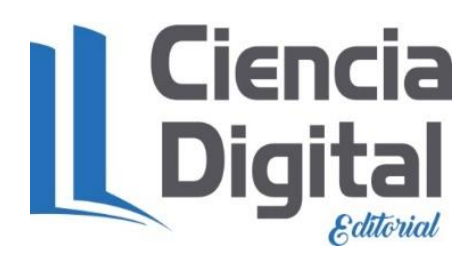

El artículo que se publica es de exclusiva responsabilidad de los autores y no necesariamente reflejan el pensamiento de la Revista Explorador Digital.

El articulo queda en propiedad de la revista y, por tanto, su publicación parcial y/o total en otro medio tiene que ser autorizado por el director o editor de la Revista Explorador Digital.
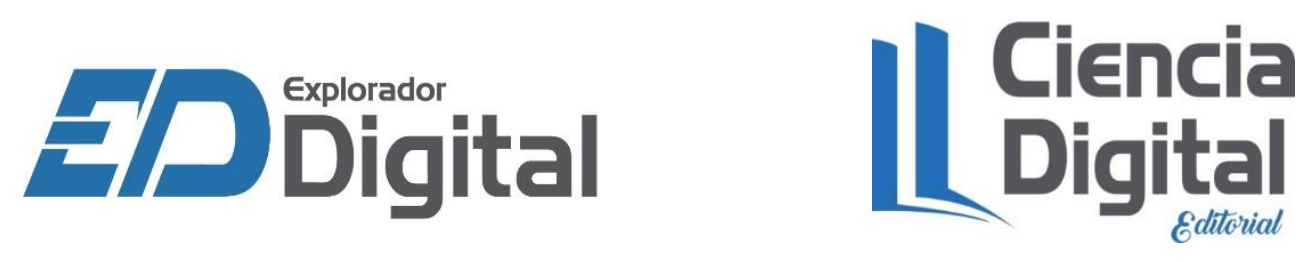